\title{
X-RAY DIFFRACTION INVESTIGATIONS OF STRUCTURE OF SILICON SINGLE CRYSTALS AFTER IRRADIATION BY HEAVY IONS
}

\author{
J. Auleytner \\ Institute of Physics, Polish Academy of Sciences \\ Al. Lotników 32/46, 02-668 Warszawa, Poland

\begin{abstract}
V.I. Khrupa, L.I. Datsenko, S.M. Krasulya and M.J. Skorokhod
Institute of Semiconductor Physics, National Academy of Sciences, Kiev, Ukraine
\end{abstract}

\begin{abstract}
Structure distortions appearing near the surfaces of crystals irradiated by high energy ions ( $\mathrm{H}, \mathrm{Kr}, \mathrm{U}$ ) accelerated till energy of several $\mathrm{MeV}$ using respectively the accelerator U-120 (Kiev, Ukraine), the heavy ions accelerators (Caen, France and Darmstadt, Germany) were investigated by means of various X-ray diffraction methods (topography and diffractometry). Nonhomogeneous distribution of lattice distortions near the surfaces of irradiated crystals were discovered using these methods in all of the samples. Besides the barrier zones where the accelerated ions stopped, the wide distorted regions situated nearer the surface were found. The fine structure of different zones, their extents as well as the level of static Debye-Waller factor were determined. The depth distribution of this factor was compared with the results obtained by using the edge contrast measurements some years ago. This permitted us to draw conclusions about some relaxation of elastic strains in the interference regions after many years.
\end{abstract}

PACS numbers: $81.40 .-\mathrm{z}, 68.35 . \mathrm{Bs}$

\section{Introduction}

The investigations [1-7] showed that the structures of silicon crystals irradiated by heavy ions with a few $\mathrm{MeV}$ energies are characterized by the different states of lattice deformations.

The known model of crystal structural changes after implantation of high energy ions assumes that along the ion track the complexes of atoms displaced from the nodal positions have been formed. Investigations of such areas show a strongly deformed amorphous layer which is separated by layers of a less damaged structure. It is evident that the radiation defects can have a considerable influence on the physical properties of silicon crystals. Therefore, it is very important, from 
the practical and scientific points of view, to obtain some quantitative information about the effects appearing during the different radiation experiments.

The aim of this paper is a quantitative analysis of the real structure of silicon crystals after irradiation by high energy ions using the X-ray topographical and other diffraction methods.

\section{Experimental}

The studied dislocation-free Si-wafers before irradiation did not reveal any prominent lattice deformation. The measurements were performed by using the Lang method. Before irradiation the surface of investigated samples was chemically polished and etched. The three series of silicon crystals were investigated. The plate from series $\mathrm{I}(t=1800 \mu \mathrm{m}$ thick) was irradiated by the $6 \mathrm{MeV} / \mathrm{n}$ protons (dose $1.3 \times 10^{17} \mathrm{i} / \mathrm{cm}^{2}, \mathrm{U}-120$, Ukraine). The thinner plate from series II $(t=200 \mu \mathrm{m})$ was irradiated by the $16 \mathrm{MeV} / \mathrm{n}$ uranium ions using the Darmstadt accelerator. The applied dose was $4.4 \times 10^{12} \mathrm{i} / \mathrm{cm}^{2}$. Series III of silicon crystals was irradiated by $\mathrm{Kr}$ ions (energy $42 \mathrm{MeV} / \mathrm{n}$, dose $3 \times 10^{13} \mathrm{i} / \mathrm{cm}^{2}$ ). Studying the plates $1-5$ from series III the deformations of structure were observed caused by the ions which were running through the four plates of $190 \mu \mathrm{m}$ thick each (samples 1-4) and they finally stopped in the last one (5) of the same thickness (Fig. 1). The details of irradiation conditions by the heavy-ion accelerator "GANIL" (France) were described with great care in Ref. [6]. The last experiment gave the possibility to separate the strongly deformed layer in the sample 5 from the less damaged regions which had appeared in the previous samples. The X-ray topographical studies were carried out for each sample [6,7].

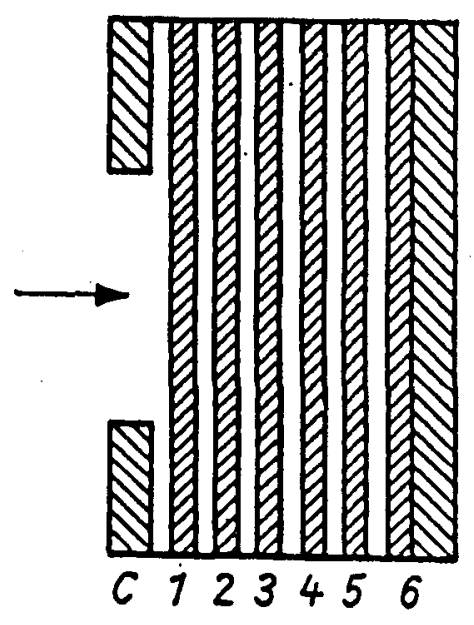

Fig. 1. Arrangement of Si plates (1-6) during implantation with Kr ions. C - copper plate.

The purpose of the special method of measurements; applied by the authors, in the present work, was to obtain the diffraction characteristics of structure deformation. This method was described in Ref. [8]. In the irradiated samples an 
increase in intensity of a diffraction peak was observed for Laue reflections (Laue case) of high orders (reflections: 440,660) or an increase in the integral reflectivity (IR) $\Delta R_{\mathrm{i}}=R_{\mathrm{i}}^{\mathrm{d}}-R_{\mathrm{i}}^{\mathrm{p}}$ comparing with the perfect crystal $\left(R_{\mathrm{i}}^{\mathrm{d}}-\right.$ the measured value, $R_{\mathrm{i}}^{\mathrm{p}}$ - the value calculated for an ideal crystal). $\Delta R_{\mathrm{i}}$ is described by the following expression:

$$
\Delta R_{\mathrm{i}}=\frac{C^{2} \pi^{2}\left|\chi_{\mathrm{rh}}\right|^{2}}{\lambda \sin 2 \theta} \frac{t}{\cos \theta} \exp \left(-\mu_{0} t / \cos \theta\right)\left[1-\exp \left(-2 L_{\boldsymbol{H}}\right)\right]
$$

where $C, \lambda, \mu, \chi_{\mathrm{rh}}$ in formula (1) are respectively: the polarization factor, the wavelength, the coefficient of photoelectric absorption and the imaginary part of the Fourier coefficient of susceptibility $\chi$ for a given reflection and $L_{\boldsymbol{H}}$ is defined later.

Using a double-crystal spectrometer for measurements of $R_{\mathrm{i}}^{\mathrm{d}}$ and formula (1) for calculations gives the possibility to determine a required value of the static Debye-Waller factor $L_{\boldsymbol{H}}$ for irradiated sample which depends not only on the vector $\boldsymbol{H}$ but also on the mean square $\langle u\rangle^{2}$ of crystal lattice atom displacement caused by the radiation defects. The value $2 L_{\boldsymbol{H}}(1)$ concerns also the volume part $\left(\rho_{0}\right)$ of disturbed lattice in which the diffuse scattering originates and, on that account the increase (IR) appears in the damaged part of the crystal, in the so-called weak absorption case $(\mu t \approx 1)$. Using the parameter $L_{\boldsymbol{H}}$ or $\rho_{0}$ one can quantitatively characterize a degree of structural perfection of the crystalline medium.

\section{Experimental data analysis}

The measurements of the $\Delta R_{\mathrm{i}}$ in silicon crystals irradiated by ions with different energies showed that in all investigated samples lattice distortions were observed. A considerable increase in $R_{\mathrm{i}}$ was visible. As an example we can present the following dependence of the relative increase $r=\Delta R_{\mathrm{i}} / R_{\mathrm{i}}^{\mathrm{p}}$ on the diffraction vector $\boldsymbol{H}$ for the reflections: 220,440,660, 880, respectively (Fig. 2). From the scheme follows that the sensitivity (IR) to the structural deformations formed during irradiation by uranium ions $(E=16 \mathrm{MeV} / \mathrm{n})$, monotonously increases together with the Laue diffraction order reaching the maximum for the reflex 880 . Except for the area where, at the depth of $160 \mu \mathrm{m}$ from the surface, the uranium ions totally agglomerated, also a less deep damaged zone was visible probably connected with the agglomeration of ions with smaller energies.

Analyzing in the case of Bragg geometry the spatial intensity distribution of diffracted beams successively reflected by different layers situated at distances $t$ from the surface of a crystal (Fig. 3), we studied the nonhomogeneous depth distribution of defects in the thick silicon plate from series $\mathrm{I}(t=1837 \mu \mathrm{m})$ irradiated by hydrogen ions. The analysis of the character of spatial distribution of diffracted beam intensity allows us to define also the integral characteristics of structure perfection of the crystal [9]. Unfortunately, it is impossible to do such an analysis, in the case when the crystal reveals simultaneously the both macro strains and local deformed regions and also in the case when the crystal is irradiated by protons. The theory of X-ray scattering in the case of crystals with complex structure deformations does not exist in this case. The increased intensity of diffraction in Fig. 3 is the result of the overlapping of effects connected with radiation defects and bending of atomic planes. 


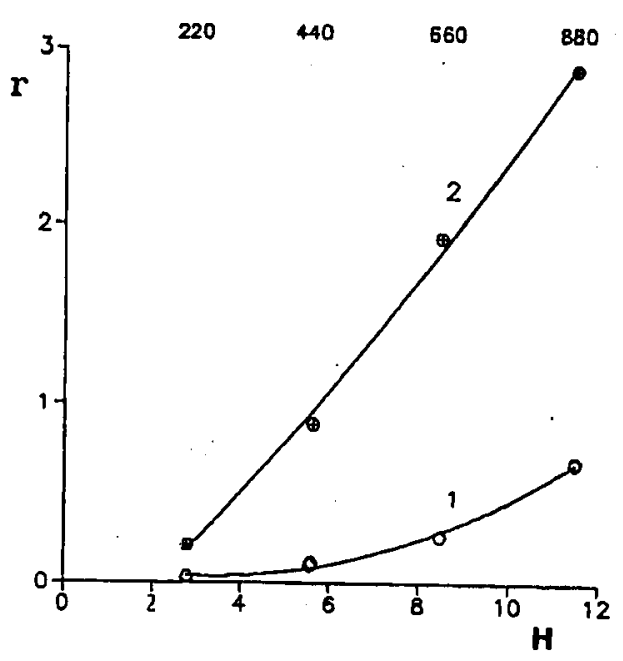

Fig. 2. The measured dependence $r$ on the diffraction vector $\boldsymbol{H}$ for the reflections $220,440,660,880$, respectively (Si crystal irradiated by uranium ions). 1 - for the non-irradiated part of the crystal, 2 - for the irradiated one.

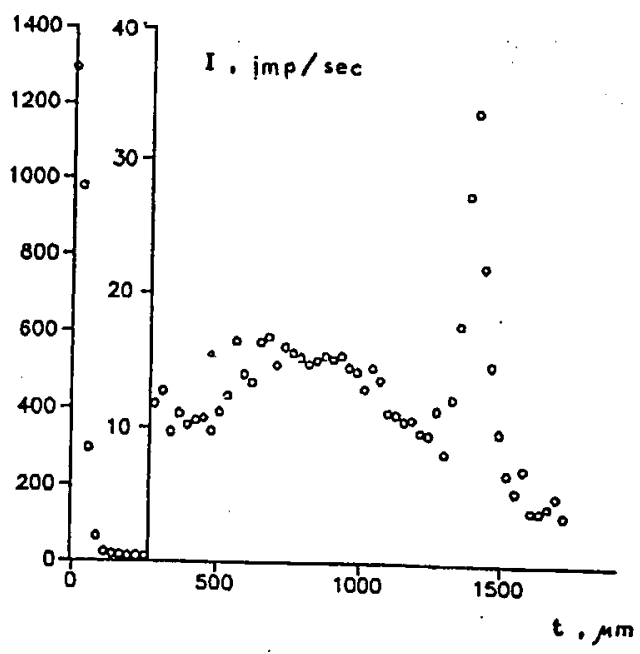

Fig. 3. The nonhomogeneous distribution of damaged structure imaged by X-ray diffraction in the case of Bragg geometry.

The exceptional possibility to define the degree of crystal structure perfection was successfully achieved after irradiation of the silicon samples 1-3 (from series III) by the high-energy krypton ions. This experiment was possible to carry out due to the ordering of several samples of the same thickness set one after an- 
other for simultaneous ion-bombardment [6]. Figure 4 presents the dependence of the integral reflectivity $R_{\mathrm{i}}^{\mathrm{d}}$ for the 660 Laue reflection and also the parameter $\rho_{0}$ on the plate number $n$. The ions ran in turn through the plates 1, 2, 3, 5. The last of them stopped in the plate 5 . The ions did not penetrate into the plate 6 and its structure perfection state is characterised by the value of the parameter $\rho_{0}=1 \times 10^{-2}$, which was close to the value of the structure of non-irradiated sample.

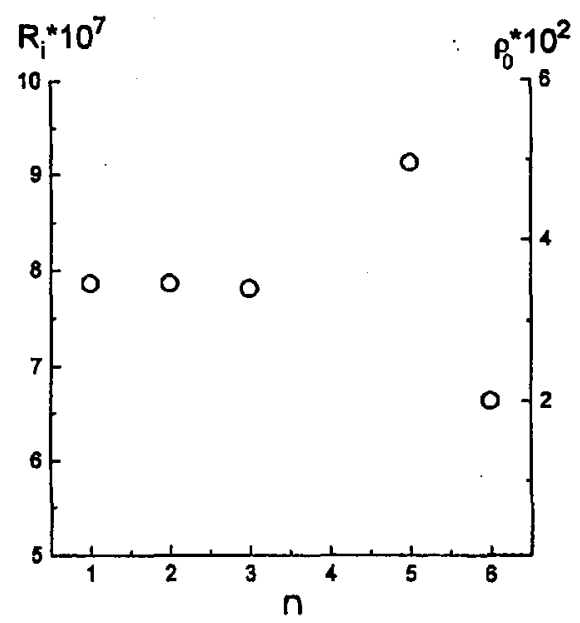

Fig. 4. The dependence of the integral reflectivity $R_{\mathrm{i}}$ for the 660 Laue reflection and the parameter $\rho_{0}$ on $n$ (plate number).

The value of $\rho_{0}$, for the plate 5 which contains the $\mathrm{Kr}$ atoms, can be treated as an effective and very conventional one because the increase in reflectivity $\Delta R_{\mathrm{i}}$ was also caused by crystal macroscopic bending.

Still smaller, despite the influence of amorphized layer, the degree of that parameter was about 1.5 times higher than the value $\rho_{0}(660)$ for samples $1-3$ without such layers $\left(\rho_{0}(660)=3 \times 10^{-2}\right)$. Because the contribution of the amorphized layer to $\rho_{0}$ has not any influence on the effect of structural changes we can consider the influence of the radiation defects which is distinctly large. Such level of $\rho$ can be usually reached in the crystals with the prominent dislocation density $\left(N_{\mathrm{g}}>10^{4} \mathrm{~cm}^{-2}\right)[8]$.

The obtained results, from our point of view, do not confirm the model of post-irradiation structural changes assuming the perfect structure of the surface layer (this model does not exist in this case). The degree of distortion level in the layers $1-3$, supposing the static Debye-Waller factor, is very substantial. Regarding the reasons of the damaged lattice in the layers 1-3 of the crystals from series III we can give the following conclusions: the part of ions with lower energies than $42 \mathrm{MeV} / \mathrm{n}$ existing in the ion-beam agglomerates into the layers $1-3$, however, the number of created tracks with displacement atoms of the matrix do not cause the effect of the overlapping the quasi perfect layers. 
Such suggestion is confirmed by the fact of existence of some diffraction effects that give the increase in intensity at the topogrammes taken by means of an asymmetric Laue-diffraction [7]. On the ground of the obtained and earlier published topogrammes [6] we can get the quantitative information testifying the partial relaxation of strains at the boundary of the implanted and non-implanted areas in sample 3 . Thus, the image of the boundary taken for the second time after 8 years is considerably reduced. Unfortunately, in Ref. [6] the measurements of the $\rho_{0}$ parameter were not carried out which might have given, at that time, a possibility to know the quantitative value of the effect.

\section{Conclusions}

The experimental results and their discussion lead to the following conclusions:

1. The application of different very sensitive diffraction methods for studying the small lattice distortions shows that the surface layer of silicon crystals implanted with high-energy ions characterizes the nonhomogeneous depth distribution of damaged regions. Besides diffraction effects coming from the amorphized layer the fluctuations of intensity from the less deep layers were observed.

2. The characteristics of structure layer perfection separating the amorphized area were obtained. There was shown that the structure was considerably deformed, however, any traces of crystal break-down were not observed. These results testify that in the non-deep surface layer the radiation defects appear, however, their main density is smaller than in the amorphized layer.

3. The comparison of the results obtained by the measurements of the integral intensity for the Laue reflection with the previously obtained topographical information on the intensity of the contrast of boundary image between implanted and non-implanted areas testifies to some relaxations of the strains during the long time of sample retaining.

\section{Acknowledgments}

This work was partially supported by the Ukrainian Committee for Science and Technology as well as partially financed by the Institute of Physics of the Polish Academy of Sciences.

\section{References}

[1] U. Bonse, M. Hart, G.H. Schwuttke, Phys. Status Solidi 33, 361 (1969).

[2] G.H. Schwuttke, K. Brock, E.E. Gardner, H.H. DeAngelis, in: Radiation Effects in Semiconductors, Ed. F.L. Vook, Plenum Press, New York 1968, p. 406.

[3] K. Wieteska, Phys. Status Solidi A 68, 179 (1981).

[4] R. Bubakova, Z. Szwid, Phys. Status Solidi 8, 105 (1965).

[5] J. Auleytner, in: Defects in Crystals, Ed. E. Mizera, World Scientific, Singapore 1988 , p. 46. 
[6] J. Auleytner, J. Bąk-Misiuk, Z. Furmanik, J. Morawiec, Cryst. Res. Technol. 23, K20 (1988).

[7] J. Auleytner, V.I. Khrupa, A. Briginets, M. Skorokhod, L. Datsenko, Cryst. Res. Technol. 30, 223 (1995).

[8] V.F. Machulin, V.I. Khrupa, Rentgenovskaya diagnostika strukturnogo sovershenstva slabo iskazennych kristalov, Naukova Dumka, Kiev 1995 (in Russian).

[9] V.I. Khrupa, I.R. Entin, Metallophisika 13, 117 (1991). 